\title{
Left Ventricle Failure and Blood Flow Estimation for
}

\section{Centrifugal Blood Pumps}

\author{
Eduardo Guy Perpétuo Bock ${ }^{1}$, Tarcísio Leão ${ }^{1}$, Jeison Fonseca ${ }^{2}$ and Aron Andrade ${ }^{2}$ \\ 1. Lab. Bioeng., Dep. Mechanics, Federal Institute of Technology in Sao Paulo IFSP, Rua Pedro Vicente, 625. Sao Paulo, SP, Brazil \\ 2. CEAC, Institute Dante Pazzanese of Cardiology, Av. Dr Dante Pazzanese, 500. Sao Paulo SP, Brazil
}

\begin{abstract}
This paper shows the blood flow control (FwC) performance to adjust rotational speed of an ICBP (implantable centrifugal blood pump) in order to provide an adequate flow to left ventricle in different patient conditions. ICBP is a totally implantable LVAD (left ventricular assist device) with ceramic bearings developed for long term circulatory assistance. FwC uses PI (proportional-integral) control to adjust rotational speed in order to provide blood flow. FwC does not use sensor for feedback, as there is an estimation system to provide blood flow measurement. Control strategy has being studied in a HCS (hybrid cardiovascular simulator) as a tool that allows the physical connection of ICBP during evaluation. In addition, HCS allows changes of some cardiovascular parameters in order to simulate specific heart disease: ejection fraction (10-25\%) and heart rate (50-110 bpm). FwC was able to adjust blood flow with steady error less than 2\%. Results demonstrated that FwC is adequate to LVAD control in different left ventricle failure conditions.
\end{abstract}

Key words: Flow control, left ventricle assist device, implantable centrifugal blood pump, heart failure, artificial organs.

\section{Introduction}

The ICBP (implantable centrifugal blood pump) is a totally implantable LVAD (left ventricular assist device) with ceramic bearings (Fig. 1). This LVAD has been developed in our laboratories for long term circulatory assistance. This LVAD was designed with original features and the studies were published previously [1].

This LVAD is composed by a continuous flow centrifugal pump, a BLDC (brushless direct current) motor, a controller to drive the motor and battery system. Centrifugal pump represents the majority of currently developed and applied research, which allows operation at lower motor speeds (approximately 2,000 rpm) than the continuous flow axial pumps (approximately 10,000 rpm). Thus, it can obtain lower rates of hemolysis, i.e., less damage to blood elements, have anatomically compatible dimensions and reach the estimated life together in

Corresponding author: Eduardo Guy Perpétuo Bock, Ph.D. Eng., associate professor, research fields: artificial organs, control, biotribology, bioengineering, biomaterials and artificial heart. support of two years [2].

The centrifugal pumps are controlled by varying the rotor (impeller) speed. ICBP successful operation depends on appropriate rotational speed control system, ensuring: (1) no reverse flow through the pump during the left ventricle diastolic phase, which usually occurs when pump speed is set too low causing flow from aorta to left ventricle through the pump; and (2) aortic valve correct opening, avoiding later valve stenosis. In order to achieve these requirements, it is necessary to control the VAD flow [3].

The goal of a VAD control is to provide a satisfactory cardiac output while sustaining an appropriate pressure perfusion. To achieve adequate control system, development has been carried out to estimate the pump parameters as VAD flow and differential pressure. Sensor is not desirable, because it can result in thrombus formation and system reliability reduction. System that detects the changes in the body's metabolic demands is one of the important objective in designing VAD control system [4].

Giridharam et al. [5] show a control strategy of maintaining a constant average pressure difference 


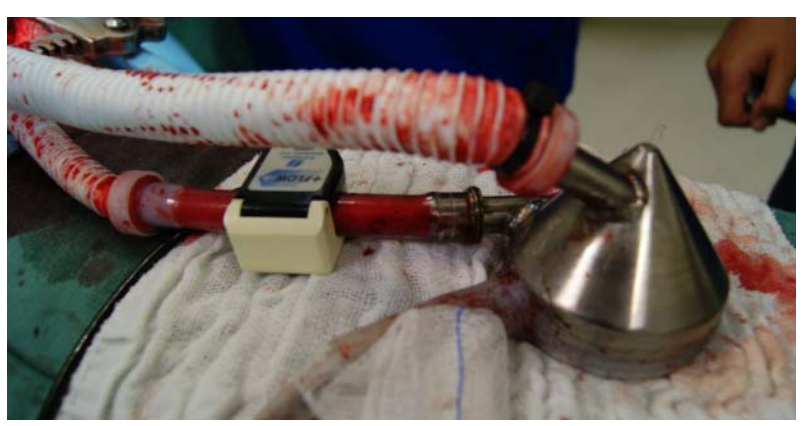

Fig. 1 ICBP during the vivo experiments.

between the pulmonary vein and aorta. Results also showed that control strategy was able to maintain total flow rate to its physiologically normal limits.

Fu and Xu [6] propose a sensorless fuzzy controller utilizing pump motor speed and current. Results indicated that proposed control can achieve required pump flow, but proportionality between pump flow and heart rate has been limited factor.

The blood flow control (FwC) adjusts rotational speed of the pump in order to provide an adequate flow to left ventricle. The FwC is part of a novel technique for automatic control of speed of VAD, which operates at harmoniously with pressure control physiological system. This technique adjusts VAD speed via FwC and fuzzy control system (FzC). Fig. 2 shows block diagram of the novel technique, where
FwC is in 2nd Layer [2]. The flow estimator is in patent process, and its error is $0.23 \pm 0.07 \mathrm{~L} / \mathrm{min}$ (confidence interval 95\%).

This paper has been divided into two main parts: the first part consists of response dynamics analysis of 1st and 2nd layers; and the second part shows FwC performance analysis. Thus, the main contribution of this paper is FwC validation under heart failure conditions.

\section{Experimental Apparatus}

To analyze system dynamics, a mock loop was used to simulate a ventricle without contraction (Fig. 3). Flow is monitored (HT110, Transonic, New York, USA) and pressures (DX2020, Biomedical Dixtal, São Paulo) [7]. Circuitry consists: $5 \mathrm{~L}$ acrylic reservoir, silicone flexible tubes $3 / 8$ " to the connection between the reservoir and pump, tourniquet to adjust flow, and a solution of distilled water (50\%) and glycerine (50\%) to simulate the viscosity of blood with hematocrit of $45 \%$, i.e., $3.2 \mathrm{mPas}$. The temperature is maintained at $25^{\circ} \mathrm{C}$ to simulate the viscosity under physiological conditions [7, 8].

To analyze FwC under heart failure condition, was used a HCS (hybrid cardiovascular simulator) (Fig. 4).

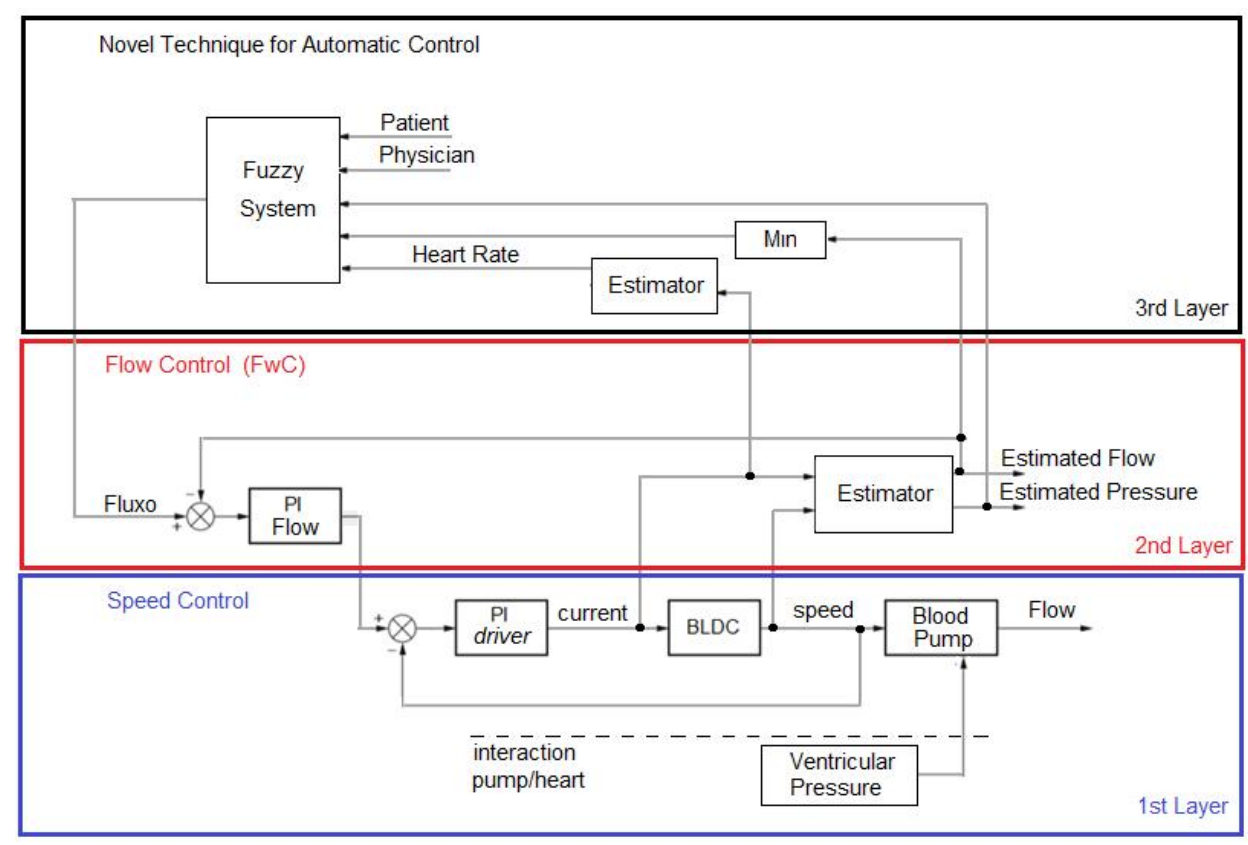

Fig. 2 Novel technique for VAD speed AUTOMATIC Control. 


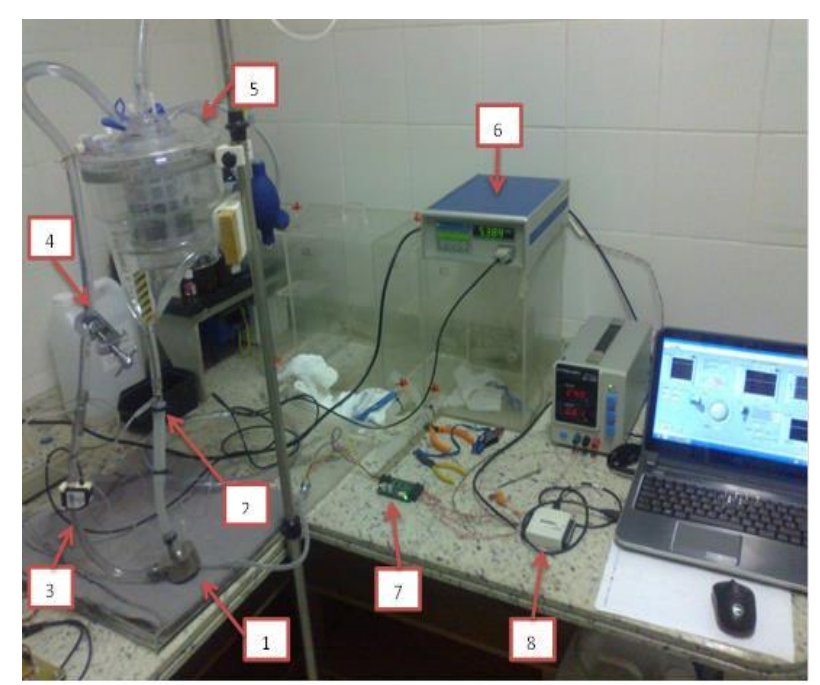

Fig. 3 A classic experimental mock loop circuit: (1) pump; (2) pressure; (3) flow; (4) tourniquet; (5) reservoir; (6) flow; (7) driver; and (8) acquisition.

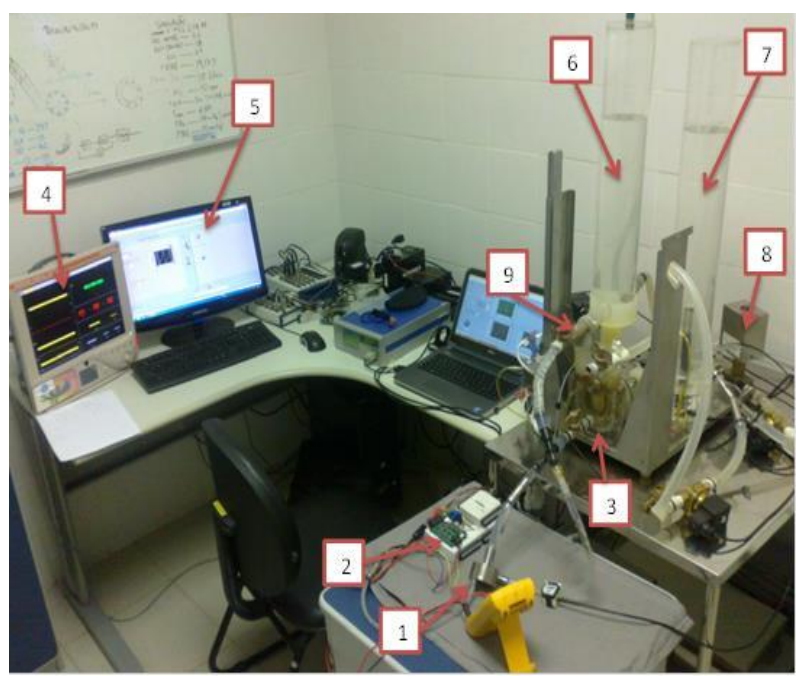

Fig. 4 HCS during experiments: (1) pump; (2) acquisition; (3) "ventricle"; (4) arterial pressure; (5) computational (right heart); (6) aorta; (7) left atrium; (8) sistemic vascular resistance; and (9) aorta.

This tool allows physical connection of pump under evaluation. In addition, HCS allows changes of some cardiovascular parameters in order to simulate specific heart disease: ejection fraction (10, 15 and 25\%) and heart rate (50, 80 and $110 \mathrm{bpm}$ ) [9].

Heart failure conditions were obtained from unpublished data from health service of Institute Dante Pazzanese of Cardiology in Sao Paulo.

FwC was implemented in Labview (2010, National Instruments, Austin, USA) and acquisition system
(USB-6009 e USB-6212, National Instruments, Austin, USA) to obtain transfer function in 1st and 2nd layers [8].

\section{Results}

Eq. (1) shows approximated TF (transfer function) (Laplace dominion) experimentally obtained (identified) $\left(G_{(s)}\right)$ at mock loop tests with pump (Fig. 3). TF input is BLDC rotational speed and TF output is VAD mean flow.

BLDC driver has minimum rotational speed, because sensorless commutation, then TF input was calculated by Eq. (2).

$$
\begin{gathered}
G_{(S)}=\frac{0.00192}{s^{2}+0.99 s+0.64} \\
R P M_{G_{(s)}}=R P M_{V A D}-513
\end{gathered}
$$

Eq. (3) shows flow control (PI) TF (Gpi $\left(\mathrm{Gi}_{(\mathrm{s})}\right)$, its input is flow error and output is reference voltage to BLDC driver. Close loop TF $\left(\mathrm{Gcl}_{(\mathrm{s})}\right)$ was calculated according to 2nd layer (FwC, Fig. 2), Eq. (4), its input is VAD flow reference and output is VAD mean flow (actual).

$$
\begin{gathered}
G p i_{(s)}=\frac{0.6 s+0.05}{s} \\
G c l_{(S)}=\frac{0.384 s+0.032}{s^{3}+0.99 s^{2}+1.024 s+0.032}
\end{gathered}
$$

Fig. 5 shows $G_{(s)}$ step response with simulated and experimental data. Fig. 6 shows $\operatorname{Gcl}_{(\mathrm{s})}$ step response with simulated and experimental data.

Approximated TF was able to represent dynamics system, morphology and steady value indicate sufficient precision to calculate mean flow. Initial conditions were different between simulated and experimental data, because flow equal zero is not possible, due to sensorless commutation.

Table 1 shows actual and estimated mean flow, with FwC adjusted to set point at $5.0 \mathrm{~L} / \mathrm{min}$, in heart failure conditions. 


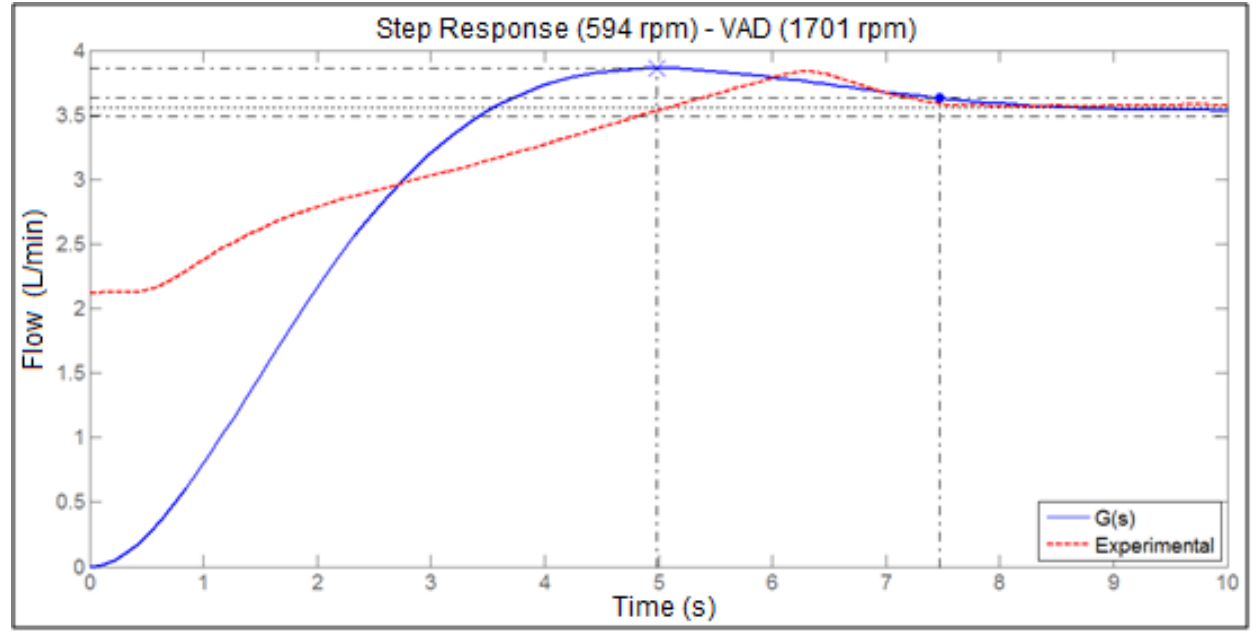

Fig. 5 Step Response $G_{(s)}$ with simulated and experimental data.

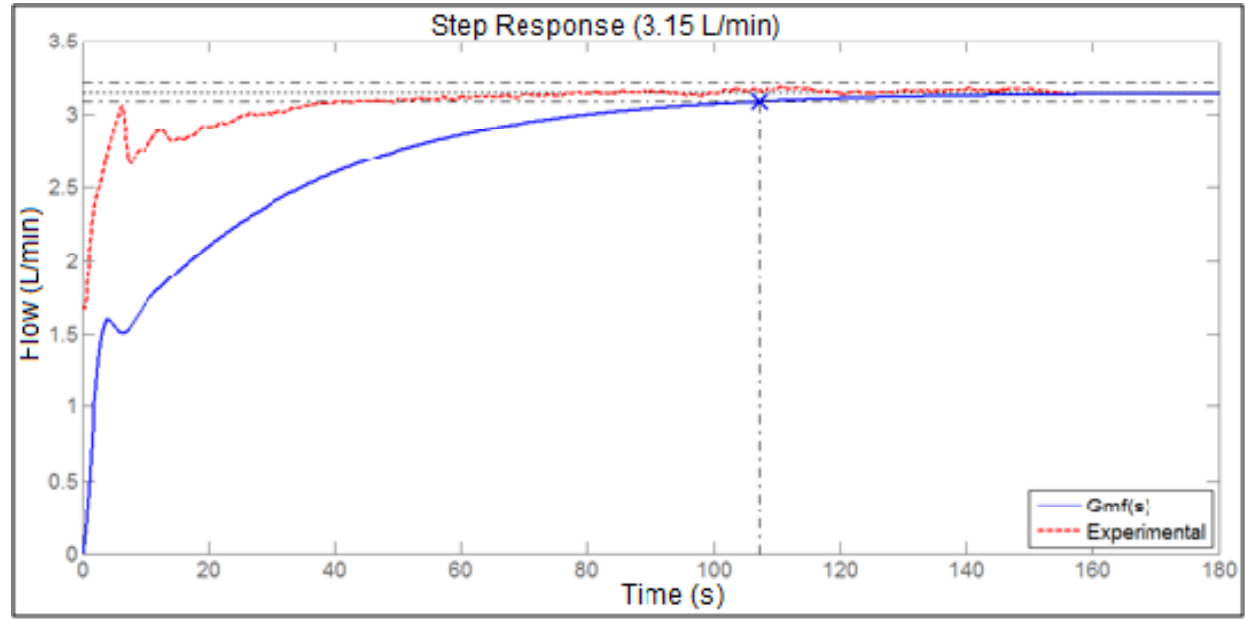

Fig. 6 Step response $\mathrm{Gcl}_{(\mathrm{s})}$ and experimental data.

Table 1 FwC performance in heart failure conditions (Set-point: $5.0 \mathrm{~L} / \mathrm{min}$ ).

\begin{tabular}{cc|cc}
\hline EF & $\begin{array}{c}\text { HR } \\
\mathbf{( b p m )}\end{array}$ & $\begin{array}{c}\text { Actual } \\
\text { Mean Flow (L/min) }\end{array}$ & $\begin{array}{c}\text { Estimated } \\
\text { Mean Flow (L/min) }\end{array}$ \\
\hline & 50 & 4.83 & 5.00 \\
& & $4.77-4.87)$ & $(4.96-5.03)$ \\
$10 \%$ & 80 & 4.85 & 5.00 \\
& & $4.80-4.92)$ & $(4.98-5.02)$ \\
& 110 & $(4.78-4.87)$ & 5.00 \\
& & 4.84 & $(4.97-5.03)$ \\
& 50 & $(4.75-4.91)$ & $(4.95-5.04)$ \\
$15 \%$ & 80 & 4.78 & 5.00 \\
& & $(4.72-4.85)$ & $(4.97-5.04)$ \\
& 110 & 4.67 & 5.00 \\
& & $(4.61-4.73)$ & $(4.96-5.03)$ \\
& 50 & $(5.05-5.32)$ & $(4.90-5.06)$ \\
$25 \%$ & 80 & $(5.05-5.30)$ & $(4.96-5.06)$ \\
& & 4.98 & 5.02 \\
& 110 & $(4.91-5.07)$ & $(4.96-5.08)$ \\
\hline
\end{tabular}

Note: EF - Ejection Fraction; HR - Heart Rate

\section{Conclusions}

Dynamic system is adequate to adjust VAD rotational speed, considering other control layers and physiological effects in simulated patients.

FwC was able to maintain mean flow at reference, even under heart rate different conditions. Steady error value is less than $2 \%$.

Future works will use the $\mathrm{FwC}$ to provide a control system that operates harmoniously with the physiological system.

\section{References}

[1] Bock, E., Ribeiro, A., Silva, M., Antunes, P., Fonseca, J., Legendre, D., Leme, J., Arruda, C., Biscegli, J., Nicolosi, D., and Andrade, A. 2008. "New Centrifugal Blood Pump 
with Dual Impeller and Double Pivot Bearing System: Wear Evaluation in Bearing System, Performance Tests, and Preliminary Hemolysis Tests.” Artificial Organs 32 (4): 329-33.

[2] Leão, T. F., Bock, E., Fonseca, J., Andrade, A., Cavalheiro, A., Uebelhart, B., Cardoso, J. R., Chabu, I., and Campo, A. 2012. "Modeling Study of an Implantable Centrifugal Blood Pump Actuator with Redundant Sensorless Control.” In Proceedings of the 44th Southeastern Symposium on System Theory, 174-8.

[3] Bock, E., Antunes, P., Leao, T., Uebelhart, B., Fonseca, J., Leme, J., Utiyama, B., da Silva, C., Cavalheiro, A., Filho, D. S., Dinkhuysen, J., Biscegli, J., Andrade, A., and Arruda, C. 2011. "Implantable Centrifugal Blood Pump with Dual Impeller and Double Pivot Bearing System: Electromechanical Actuator, Prototyping, and Anatomical Studies.” Artificial Organs 35 (5): 437-42.

[4] AlOmari, A., Savkin, A. V., Stevens, M., Mason, D. G., Timms, D. L., Salamonsen, R. F., Lovell, N. H. 2013. "Developments in Control Systems for Rotary Left Ventricular Assist Devices for Heart Failure Patients: A Review.” Physiological Measurement 34 (1): R1-27.

[5] Giridharan, G. A., and Skliar, M. 2006. "Physiological
Control of Blood Pumps Using Intrinsic Pump Parameters: A Computer Simulation Study.” Artificial Organs 30 (4): 301-7.

[6] Fu, M., and Xu, L. 2000. "Computer Simulation of Sensorless Fuzzy Control of a Rotary Blood Pump to Assure Normal Physiology.” ASAIO Journal 46 (3): 273-8.

[7] Leme, J., Fonseca, J., Bock, E., da Silva, C., da Silva, B. U., Dos Santos, A. E., Dinkhuysen, J., Andrade, A., and Biscegli, J. F. 2011. “A New Model of Centrifugal Blood Pump for Cardiopulmonary Bypass: Design Improvement, Performance, and Hemolysis Tests.” Artificial Organs 35 (5): 443-7.

[8] Fonseca, J., Andrade, A., Nicolosi, D. E., Biscegli, J. F., Leme, J., Legendre, D., Bock, E., and Lucchi, J. C. 2011. “Cardiovascular Simulator Improvement: Pressure versus Volume Loop Assessment.” Artificial Organs 35 (5): 454-8.

[9] Leão, T., Fonseca, J., Bock, E., Utiyama, B., Drigo, E., Sá, R. C. L., Leme, J., Nicolosi, D., Biscegli, J., and Andrade, A. 2015. "Development of Rotational Automatic Control Method to an Implantable Centrifugal Blood Pump.” Presented at the ASAIO 61st Annual Conference, Chicago, USA. 